\title{
Clinicopathological study of gastric carcinoma in high- and low-mortality countries: Comparison between Japan and the United States
}

\author{
Michio Maruyama ${ }^{1-3}$, Kimiya Takeshita ${ }^{2}$, Mitsuo Endo ${ }^{2}$, Mark Deakin ${ }^{3}$, and AR Moossa ${ }^{3}$ \\ ${ }^{1}$ Department of Surgery, Tokyo Metropolitan Ohkubo Hospital, 2-44-1, Kabukicho, Shinjuku-ku, Tokyo 160, Japan \\ ${ }^{2}$ First Department of Surgery, Tokyo Medical and Dental University, 1-5-45, Yushima, Bunkyo-ku, Tokyo 113, Japan \\ ${ }^{3}$ Department Of Surgery, UCSD Medical Center, University California, San Diego, 225 Dickinson Street, San Diego, CA 92103, USA
}

\begin{abstract}
:
Background. Gastric carcinoma is a tumor that shows marked differences in geographic distribution. The incidence of the gastric carcinoma has been falling dramatically in Western countries. In sharp contrast, the incidence in Japan is still quite high.

Methods. A comparative clinicopathological study was performed, examining gastric carcinomas in 978 Japanese patients (Tokyo) and 157 United States patients (San Diego), that had been surgically resected over the 11-year period 1980-1991.

Results. The median age of US the patients (64.1 years) was higher than that of the Japanese patients (59.0 years). There was no difference in the sex ratios. Forty-five of the Japanese patients had early gastric cancer, while this occurred in $10 \%$ of the US patients. In terms of tumor location, $47 \%$ were in the upper portion of the stomach in the US patients, while, in contrast, this proportion in Japanese patients was $23 \%$. In association with the marked difference in tumor location, $36.5 \%$ of the US patients underwent operations with the thoraco-abdominal approach, while this approach was used in $7.5 \%$ of the Japanese patients. The distribution of histological types was similar in the two countries' samples. There was also no significant difference in the distribution of the histological types in the same age groups in the two countries' samples. The rate of the well differentiated type increased with age while the rate of poorly differentiated type decreased. In US patients, $35.8 \%$ had marked neural invasion, while this occurred in $8.5 \%$ of Japanese patients.

Conclusion. This study revealed marked differences in the features of gastric cancer between the US and Japan in terms of tumor location and surgical procedures.
\end{abstract}

Key words: gastric carcinoma, gastric cancer, epidemiology, histological type of gastric carcinoma, international comparison

Offprint requests to: M. Maruyama Received for publication on February 18, 1998; accepted on May 25, 1998

\section{Introduction}

Gastric carcinoma is a tumor that shows marked differences in geographic distribution [1-5]. The incidence of gastric carcinoma has been falling dramatically in Western countries (e.g., Western Europe, Australia, the United States, Canada). Over the past 50 years, both the incidence and the mortality in the US have decreased, by $70 \%$ for men and by $80 \%$ for women [1]. In sharp contrast, the incidence of gastric cancer in Japan, Singapore, China, Israel, Finland, Colombia, and Chile is quite high. Gastric carcinoma is still one of the most common cancers worldwide. In Japan, this tumor is the leading cause of carcinoma death. More than a quarter of a century ago, international studies comparing features of gastric cancer in Japan and the US showed there were no major differences in the nature of gastric cancer at that time $[1,5]$. Over the past three decades features of gastric cancer have greatly changed in both countries. In Japan early gastric carcinoma is now common. In the US, the incidence of gastric cancer has been falling. In this study we compared the clinicopathological features of gastric carcinoma in Japanese and US samples and found changes in the nature of gastric cancer in the two, countries' samples.

\section{Patients and methods}

\section{Patients}

We examined the clinicopathological characteristics of 978 gastric carcinomas resected in the period 1980-1991 at the First Department of Surgery, Tokyo Medical and Dental University (Tokyo, Japan), and 157 gastric carcinomas surgically removed in the same period (19801991) at three major hospitals in San Diego, US (UCSD Medical Center, Scripps Green Hospital, and VA Hospital, all in San Diego). These four hospitals are 
major tertiary centers in Tokyo and the San Diego area. Of the San Diego patients, 114 were white; 22, Hispanics; 5, Orientals; 5, blacks; and 10, not described.

\section{Definition of gastric carcinoma}

Gastric carcinoma was defined as cancer located mainly in the stomach on the basis of findings of the resected specimens. In Western countries, in particular, significant numbers of cancers exist around the esophagogastric junction (EGJ), for example EGJ carcinoma and esophageal adenocarcinoma. In some instances it is difficult to classify tumors around the EGJ as gastric or esophageal cancer before resection. In this study we adopted a strict definition of gastric carcinoma especially for tumors around the EGJ. Tumors, whose centers were just on the EGJ or inside the stomach were classified as gastric carcinoma. But tumors whose centers were on the esophageal side beyond the EGJ were excluded from gastric carcinomas.

All the specimens from Japan and the US were studied in detail under the same protocol by a single clinical pathologist who was unaware of their source.

\section{Gross findings}

Gross findings, in terms of location, size, and gross type, were checked.

Location of the tumor. The stomach was separated into upper, middle, and lower portions by drawing lines between the corresponding trisecting position on the greater and lesser curvature of the resected specimen. "Location of the tumor" is the location of the main portion of the tumor.

Gross type. Tumors were classified into six gross types based on visual inspection, according to the Rules of the Japanese Gastric Cancer Association. Type 0, Superficial gastric cancer which appeared confined to mucosa or submucosa. Type 1, Protruding and localized advanced tumor. Type 2, Ulcerated and localized advanced tumor. Type 3, Ulcerative tumor with infiltrative growth pattern. Type 4, Diffuse carcinoma. Type 5, Tumors not included under any of the above types.

\section{Microscopic findings}

Microscopic findings were examined in detail under a light microscope with H\&E-stained slides.

Histological type. Tumors were assigned to four histological types. The classification was based on the predominant histological pattern. 1, Well differentiated type: carcinoma with fully developed glandular, tubular or papillary pattern. The tubular or acinar glands were lined with cylindrical or cuboidal epithelium. 2, Moder- ately differentiated type: carcinoma with less well differentiated gland spaces. Carcinoma showed small nests with glandular arrangement. 3, Poorly differentiated type: carcinoma did not show any glandular pattern. This type contained diffuse carcinoma and signet ring cell carcinoma. 4, Mucinous type: carcinoma was characterized by the presence of extracellular mucin.

Histological growth pattern. Tumors were classified into three groups, medullary, intermediate and scirrhous.

Depth of cancer invasion. Depth of invasion was classified into four groups according to tumor infiltration of the gastric wall: mucosa (m), submucosa (sm), muscularis propria ( $\mathrm{mp}$ ), and cancer which invaded beyond the muscularis propria (s).

Neural invasion. For the examination of histological neural invasion, 280 of the Japanese specimens were chosen at random and all US specimens were examined. Some of the specimens were stained with anti-S100 protein monoclonal antibody, using the peroxidaseantiperoxidase method. Tumor were classified into two groups, neural invasion $(-)$ and $(+)$. Neural invasion $(+)$ tumors had marked neural invasion under the light microscope, and carcinoma cells had infiltrated along nerve bundles or the perineural space.

Statistical analyses were done with the $t$-test or $\chi^{2}$ test of these data.

\section{Results}

Age and sex

Comparison of the patients median age showed a significant difference between the two countries' samples $(P<0.001)$. San Diego patients (aged 64.1 years) were older than Tokyo patients (59.0 years) (Table 1). Sex ratios were not different between the two cities, and in both males were predominant (Tokyo; 2.3:1, San Diego; 3.4:1) (Table 1).

\section{Gross findings}

Location of the tumors. The proportion of tumors in the upper portion was $47 \%$ in the US; in sharp contrast, that in Japan was $23 \%$ (Table 2). The proportions of middle portion tumors was 19\% in the US and 39\% in Japan. US patients showed a significantly higher proportion of

Table 1. Age and sex of gastric cancer patients in Japan and United States samples

\begin{tabular}{lccc}
\hline & Japan & US & \\
\hline Age & $59.0 \pm 12.3$ & $64.1 \pm 12.6$ & $P<0.01$ \\
Sex $(\mathrm{M} / \mathrm{F})$ & $2.3: 1$ & $3.4: 1$ & NS \\
\hline
\end{tabular}

M, male; F, femald 
Table 2. Location of gastric carcinomas according to portion of the stomach in Japan and US samples

\begin{tabular}{lccc}
\hline & Japan & US & \\
\hline Location & $n(\%)$ & $n(\%)$ & \\
Upper & $225(23)$ & $70(47)$ & \\
Middle & $390(39)$ & $30(19)$ & \\
Lower & $314(32)$ & $41(26)$ & \\
Entire & $51(5.2)$ & $13(8.0)$ & \\
Total & $980(100)$ & $157(100)$ & $P=0.0009$ \\
Gross tympe & $n(\%)$ & $n(\%)$ & \\
Type 0 & $424(45.2)$ & $15(9.6)$ & \\
Type 1 & $24(2.6)$ & $12(7.7)$ & \\
Type 2 & $118(12.7)$ & $32(20.5)$ & \\
Type 3 & $248(26.3)$ & $64(41.0)$ & \\
Type 4 & $63(6.7)$ & $29(18.6)$ & \\
Type 5 & $63(6.7)$ & $4(2.6)$ & \\
Total & $939(100)$ & $156(100)$ & $P<0.0001$ \\
Size (cm) & $5.36 \pm 3.76$ & $6.44 \pm 3.52$ & $P<0.0001$ \\
& $7.67 \pm 3.87 *$ & $6.94 \pm 3.49 *$ & NS \\
\hline
\end{tabular}

* Median size of "s" tumors (i.e. those that invaded beyond the muscularis propria)

upper portion cancers and significantly lower proportion of middle portion cancers than in Japan $(P=$ 0.0009). In Japanese patients, the proportion of upper and lower portion carcinomas increased with age (Fig. 1), while middle portion carcinomas decreased. In the US patients, the proportion of upper portion tumors was predominant in all age groups, with the proportion in the age group 56-65 years high, at 56.4\% (Fig. 1). The proportion of lower portion tumors increased with age in the US, similarly to findings in the Japanese sample. Statistical analysis of the relationship between age and portion showed between each counterpart age group in Japan and the US, with significant differences in the groups under age 55 and over age 66 $(P<0.001)$.

Gross type of tumor. There were significant differences between Japan and the US in gross type of tumor $(P<$ 0.0001 ); in Japanese, $45 \%$ of the tumors were type- 0 (i.e., early gastric cancer), while in the US patients, fewer than $10 \%$ were this type (Table 2 ). When type 0 tumors were excluded, the distribution of gross types showed no significant difference between the two countries $(P=0.0707)$.

Tumor size. The median size of the tumors in Japanese was $5.36 \mathrm{~cm}$, while that in the US sample was $6.44 \mathrm{~cm}$ (Table 2). In the Japanese sample, there was a much smaller mean size $(P<0.0001)$ because of the high proportion of early-stage gastric carcinoma. The median size of "s" tumors (invasion beyond the muscularis propria), was not significantly different between the two countries (Japan, $7.67 \mathrm{~cm}$; US, $6.94 \mathrm{~cm}$ ).
U.S. age-portion

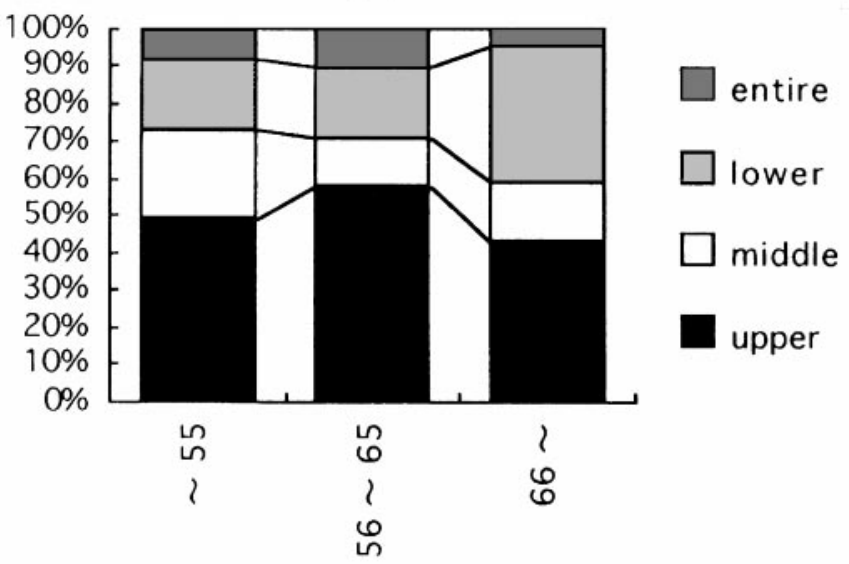

Japan age-portion



Fig. 1. Relationship between age and portion of stowach in which tumor was located

\section{Microscopic findings}

Histological type. There were no significant differences in the distribution of the four histological types of tumors between the Japanese and US samples (Table 3). Concerning the relationship between location and histological type of tumor, there was no significant difference in the proportions of the histological types in each portion of the stomach between the two countries' samples (Fig. 2). There was also no difference in the proportion of each histological type between the US and Japanese samples from the same age groups (Fig. $3)$. Poorly differentiated type was predominant under the age of 55 years in both countries $(64.9 \%$ in the US and $60.7 \%$ in Japan), while the proportion of well differentiated type in this age group was $18.9 \%$ in the US and $18.5 \%$ in the Japanese sample. The proportion of well differentiated type increased and that of poorly differentiated type decreased with age. In the over 66 age group, the percentage of poorly differentiated type 
Table 3. Histological features of gastric carcinomas in Japan and US samples

\begin{tabular}{|c|c|c|c|}
\hline & Japan & US & \\
\hline Histological type & $n(\%)$ & $n(\%)$ & \\
\hline Well differentiated & $297(31.3)$ & $47(29.9)$ & \\
\hline Moderately differentiated & $201(21.2)$ & $29(18.5)$ & \\
\hline Poorly differentiated & $416(43.8)$ & $73(46.5)$ & \\
\hline Mucinous & $23(2.4)$ & $9(5.7)$ & \\
\hline Total & $937(100)$ & $158(100)$ & $P=0.5180$ \\
\hline $\begin{array}{l}\text { Depth of invasion } \\
\text { m (mucosa) } \\
\text { sm (submucosa) } \\
\text { mp (muscularis propria) } \\
\text { s (beyond mp) }\end{array}$ & $\begin{array}{c}n(\%) \\
226(24.3) \\
207(22.3) \\
101(10.9) \\
394(42.6)\end{array}$ & $\begin{aligned} & n(\%) \\
& 5(3.2) \\
& 10(6.4) \\
& 9(5.7) \\
& 133(84.7)\end{aligned}$ & \\
\hline Total & $928(100)$ & $157(100)$ & $P<0.0001$ \\
\hline $\begin{array}{l}\text { Growth pattern } \\
\text { Medullary } \\
\text { Intermediate } \\
\text { Scirrhous }\end{array}$ & $\begin{array}{c}n(\%) \\
167(26.3) \\
338(53.1) \\
131(20.6)\end{array}$ & $\begin{array}{c}n(\%) \\
38(25.3) \\
71(47.3) \\
41(27.3)\end{array}$ & \\
\hline Total & $636(100)$ & $150(100)$ & $P=0.5699$ \\
\hline $\begin{array}{l}\text { Lymph node metastasis } \\
\text { m (mucosa) } \\
\text { sm (submucosa) } \\
\text { mp (muscularis propria) } \\
\text { s (beyond mp) }\end{array}$ & $\begin{array}{c}n / \text { Total } n(\%) \\
4 / 219(1.8) \\
21 / 199(10.6) \\
38 / 97(39.2) \\
270 / 346(78.0)\end{array}$ & $\begin{array}{l}n / \text { Total } n(\%) \\
0 / 4(0) \\
4 / 10(40.0) \\
2 / 8(25.0) \\
105 / 128(82.0)\end{array}$ & \\
\hline Total & 333/861 (38.7) & $111 / 150(74.0)$ & $P=0.0001$ \\
\hline $\begin{array}{l}\text { Neural invasion* } \\
\text { Well differentiated } \\
\text { Moderately differentiated } \\
\text { Poorly differentiated } \\
\text { Mucinous }\end{array}$ & $\begin{array}{l}n / \text { Total } n(\%) \\
0 / 18(0) \\
4 / 33(12.1) \\
6 / 73(7.9) \\
1 / 6(16.7) \\
11 / 130(83)\end{array}$ & $\begin{array}{l}n / \text { Total } n(\%) \\
3 / 29(10.3) \\
9 / 27(33.3) \\
36 / 71(50.7) \\
0 / 7(0) \\
48 / 134(358)\end{array}$ & \\
\hline Total & 11/130 (8.3) & 48/134 (35.8) & $P<0.0001$ \\
\hline
\end{tabular}

*Tumor invasion beyond muscularis propria

was $37.2 \%$ in the US sample and $32.5 \%$ in the Japanese, while the proportion of well differentiated type was $38.5 \%$ in the US and $43.1 \%$ in the Japanese. There was a significant difference between the under 55 and 56 to 65 age groups and between the under 55 and the over 66 age groups in Japan $(P<0.001)$.

Histological growth pattern. There were no significant differences in histological growth pattern between the US and Japanese samples (Table 3).

Lymph node metastasis. There were no significant differences in lymph node metastasis at each depth of invasion between the two countries' samples (Table $3)$.

Neural invasion. Findings for neural invasion were significantly different in the two countries' sample $(P<$ 0.0001). In examined samples, neural invasion was observed in s (depth) tumors, which invaded beyond the muscularis propria, while no marked neural invasion was observed among tumors confined to the mucosa, submucosa or muscularis propria (Fig. 4). Of the 133 Japanese "s" tumors, 11 (8.3\%) showed marked neural invasion (Table 3). In sharp contrast, of 134 US "s" tumors $48(35.8 \%)$ showed marked neural invasion (Table 3), although the median size of the "s" tumors was not significantly different in the two countries' samples (Table 2). Both moderately differentiated and poorly differentiated types of tumors were likely to show neural invasion in the samples of both countries. In the Japanese sample, $12.1 \%$ of the moderately differentiated type, and $7.9 \%$ of the poorly differentiated showed neural invasion. In the US sample $33.3 \%$ of the moderately differentiated type and $50.7 \%$ of the poorly differentiated type showed neural invasion.

\section{Type of operations}

There was a marked difference in types of operations for gastric cancers in the two countries' samples (Table 
U.S. location and histology

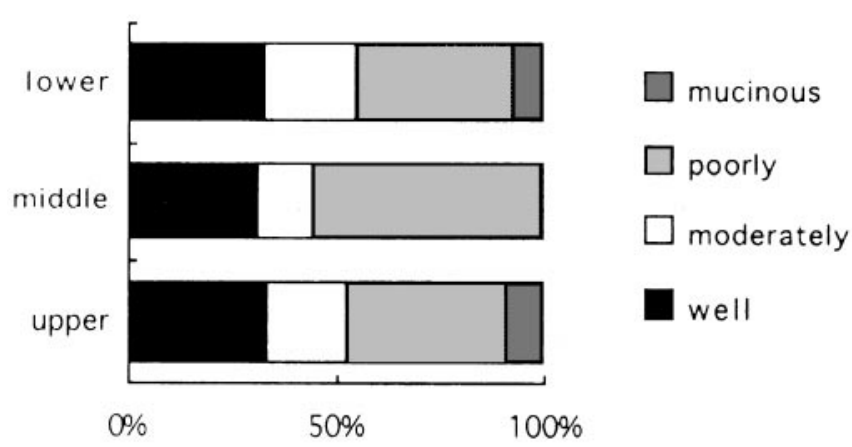

Japan location and histology

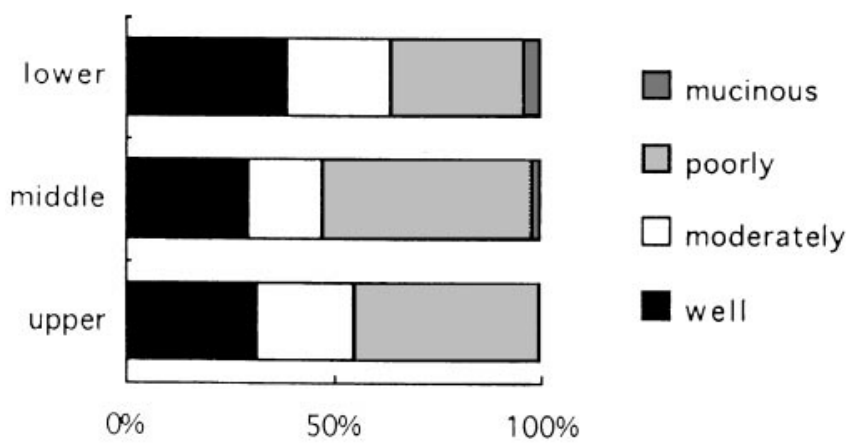

Fig. 2. Relationship between location of tumor and histological type

4) $(P=0.0001)$. Fifty-seven $(36.5 \%)$ of the US patients had esophago-gastrectomy or total gastrectomy + distal esophagectomy, most associated with the thoracoabdominal approach. In sharp contrast, only $7.2 \%$ of the Japanese patients had the same type of operation; $601(61.6 \%)$ of the Japanese patients underwent distal or subtotal gastrectomy, while $53(34.0 \%)$ of the US patients underwent the same operation.

\section{Discussion}

Gastric carcinoma is the most common carcinoma in Japan, and is the most common cause of cancer death in both men and women. However, gastric carcinoma mortality rates have been decreasing slightly in Japan, one reason being that many early-stage carcinomas are being found through mass screening checks [6]. Currently, the incidence of early gastric carcinoma (confined to the mucosa or submucosa) is more than $40 \%$ of all gastric carcinomas in most hospitals in Japan. The overall 5-year survival rate for gastric cancer patients who underwent resections in Japanese facilities is $68 \%$. In contrast to Japan, the incidence of gastric carcinoma in the US has been falling dramatically in the past five
U.S. age-histhology

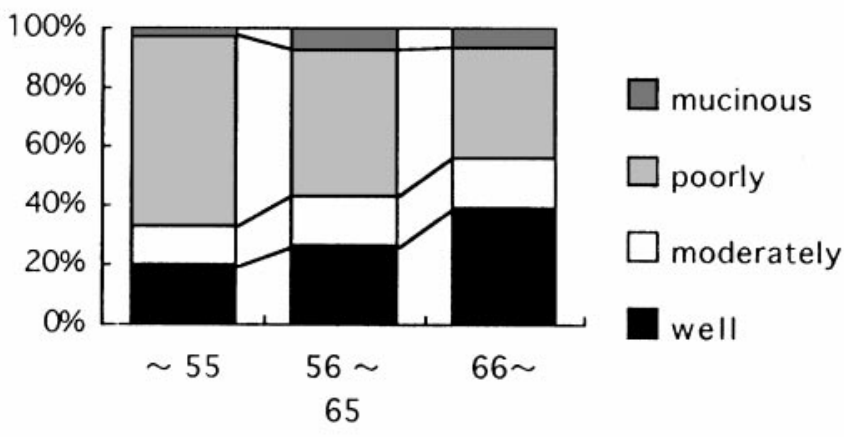

Japan age-histhology

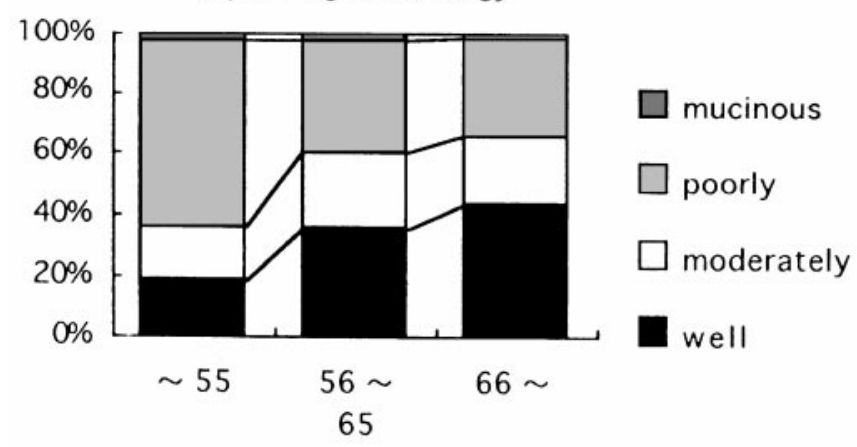

Fig. 3. Relationship between patient age and histological type of tumor

decades. Other western countries also have a low incidence of gastric cancer. Because of this low incidence, there are no mass screening programs in western countries. Most patients had advanced cancers when diagnosed [7-9], and the survival rate in western countries was low, $5 \%-20 \%[7,10,11]$. Previous reports show the mean age and sex ratios of gastric cancer patients are similar to our data in this study, indicating that our population represents the general characteristics of gastric cancer in both the US and Japan $[2,11,12]$.

Articles published 30-40 years ago showed no differences in the anatomical location of gastric carcinoma worldwide, with $60 \%-70 \%$ of the gastric carcinomas located in the lower two-thirds of the stomach in both of Asian and western countries [4,13-16]. However, in this study, almost $50 \%$ of the tumors in the US samples were located in the upper third of the stomach. In sharp contrast, the proportion of the tumors in the upper third of the stomach in the Japanese sample was 23\%. Further, this proportion in Tokyo was much higher than that in other hospitals in Japan, as the authors' hospital in Tokyo treated many esophageal and EGJ carcinomas. The proportion of upper portion tumors in most Japanese hospitals was found to be less than 20\% [9]. In the past 30-40 years, the proportion of lower portion 

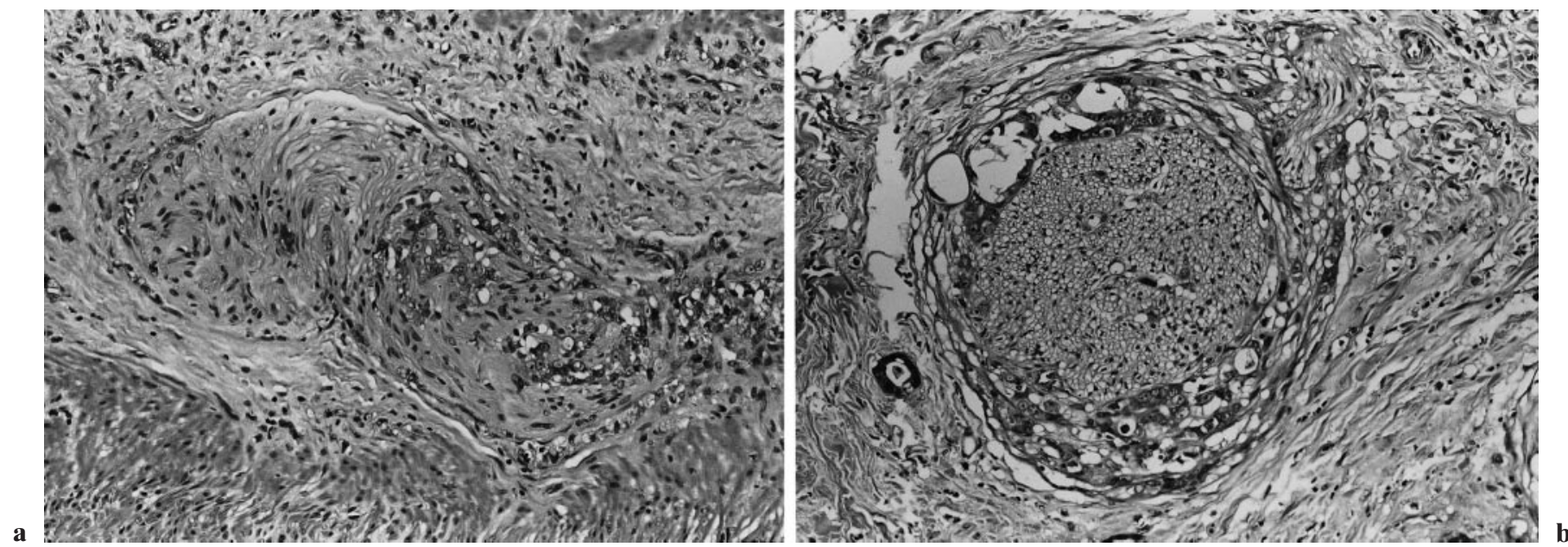

Fig. 4a,b. Neural invasion of gastric carcinoma. a Signet ring cell cancer cells infiltrating along the submucosal nerve bundle b Carcinoma cells invading the perineural space, so-called perineural invasion $\mathbf{a}, \mathbf{b} ; \mathrm{H} \& \mathrm{E} \times 200$

Table 4. Type of operations for gastric cancer in Japanese and US patients

\begin{tabular}{lrr}
\hline & Japan & US \\
\cline { 2 - 3 } Operation & $n(\%)$ & $n(\%)$ \\
\hline $\begin{array}{l}\text { Esophago-gastrectomy or } \\
\quad \text { total gastrectomy }+\end{array}$ & $70(7.2)$ & $57(36.5)$ \\
$\quad$ distal esophagectomy) & & \\
$\begin{array}{l}\text { Total gastrectomy } \\
\text { Distal gastrectomy or }\end{array}$ & $283(29.0)$ & $38(24.4)$ \\
subtotal gastrectomy & $601(61.6)$ & $53(34.0)$ \\
$\begin{array}{l}\text { Proximal gastrectomy } \\
\text { Other }\end{array}$ & $8(0.8)$ & $4(2.6)$ \\
Total & $13(1.3)$ & $4(2.6)$ \\
\hline
\end{tabular}

$P<0.0001$

tumors of the stomach has been decreasing in western countries, but it has not changed in Japan. In Japan the proportion of lower and upper portion carcinomas increased with age, while the proportion of middle portion tumors, predominant in the under 55 age group $(50.8 \%)$, gradually decreased. In the US sample upper portion carcinoma was predominant at all ages, particularly so at age $56-65$ years $(56.4 \%)$. The proportion of lower portion tumors in the US sample increased with age.

There was a marked difference between the two countries in types of operation for gastric carcinoma. The differences in tumor location led to the different distributions of operation methods; $36.5 \%$ of US patients underwent esophago-gastrectomy or total gastrectomy + distal esophagectomy, most associated with the thoraco-abdominal approach. In contrast, only $7.2 \%$ of patients in Japan needed the thoracoabdominal approach. Instead, distal or subtotal gastrectomies were performed for $61.6 \%$ of the gastric tumors in the Japanese patients, while this operative approach was taken in $34 \%$ of patients in the US sample. Recent trends in gastric carcinoma in western countries are that the incidence of distal lesion has been diminishing and the incidence of cardia and EGJ carcinomas has been increasing [10-12,17,18]. Features of gastric cancer have been changing sharply in the past $30-40$ years in the US. It is possible that different underlying conditions influence the different incidences of the tumor in the upper third of the stomach [19-21]. In fact, in this present study, most patients with cardia or EGJ tumors in the US had had symptoms related to chronic reflex and hiatus hernia.

About 30 years ago, in an international comparative study of chronic gastritis [22], intestinal metaplasia was found in $80 \%$ of Japanese subjects over the age of 40 years, while the incidence among Americans of the same age group was only $30 \%$. Most researchers suspected that this different rate influenced the distribution of the histological types of the gastric cancer, in the light of the hypothesis proposed by Nakamura et al. [23] that the intestinal type of gastric cancer was derived from intestinal metaplasia and the diffuse type from the original mucosa of the stomach. From this point of view, one would expect that Japanese, who had a high incidence of intestinal metaplasia, would have a higher incidence of the intestinal type of tumor than Americans, who had a low incidence of intestinal metaplasia. But this study revealed no significant difference in the distribution of the histological types of the gastric cancers between the samples of the two countries. Kubo [2] also 
noted a similarly unexpected result, that gastric carcinoma in Japanese and American populations showed the same distribution of histological types about 25 years ago. The incidence of well differentiated type increased with age in both countries, while, in contrast, the incidence of poorly differentiated type decreased. This phenomenon was anticipated, from the hypothesis that the area of intestinal metaplasia in the gastric mucosa became wider with age [23].

Neural invasion was recognized in tumors which had invaded beyond the muscularis propria in the samples of both countries, with $35.8 \%$ tumors in the US sample with a depth of invasion beyond the muscular is propria showing neural invasion, while this occurred in only $8.3 \%$ of the Japanese counterparts. This finding suggested that the biological behavior in regard to invasion to the nerve was different between the US and Japanese samples.

In the pancreatic carcinoma, nerve invasion is the major pathway in the retroperitoneal direction [24]. In rectal carcinoma, neural invasion has a strong relationship with local recurrence of the tumor [25]. To date, there has been no evidence showing whether histological neural invasion of gastric cancer results in different clinical characteristics. The authors will be investigating this aspect in the next step of the study.

Acknowledgments. The authors express their profound appreciation to Dr. R. Moreki and Dr. K. Miyai at UCSD Medical Center; Dr. B. Stabile and Dr. R. Astlita at VA Hospital, San Diego; and Dr. M. Elliott at Scripps Green Hospital, all of whom kindly allowed us access to the histological specimens and gave great support to this study.

\section{References}

1. Frank-Stromborg M. The epidemiology and primary prevention of gastric and esophageal cancer. Cancer Nursing 1989;12:5364.

2. Kubo T. Histologic appearance of gastric carcinoma in high and low mortality countries: Comparison between Kyushu, Japan and Minnesota, USA. Cancer 1971;28:726-34.

3. Correa P, Sasano N, Stemmermann GN, Haenszel W. Pathology of gastric carcinoma in the Japanese population: Comparisons between Miyagi Prefecture, Japan and Hawaii. Natl Cancer Inst 1973;51:1449-56.
4. Kubo T. Geographical pathology of gastric carcinoma. Acta Pathol Jap 1974;24:465-79.

5. Nomura A. Stomach cancer. In: Schottenfeld D, Fraumeni J, editors. Cancer epidemiology and prevention. Philadelphia: WB Saunders; 1982; p. 624-37.

6. Kidokoro T. Clinical aspects of gastric cancer. Tokyo: Health Shuppan, 1983; p. 47-9.

7. Fielding JWL, Roginski C, Ellis DJ, Jones BG, Powell J, Waterhouse JA, et al: Clinicopathological staging of gastric cancer. Br J Surg 1984;71:677-80.

8. Green PHR, O'Toole KM, Solonim D, Wang T, Weg A. Increasing incidence and excellent survival of patients with early gastric cancer: Experience in a United States Medical Center. Am J Med 1988;85:85-90.

9. Nishi M, Ohota K, Ishihara S, Nakajima S. Treatment results of gastric cancer. (in Japanese) Gastroenterological Surgery 1990;15: 716-21.

10. Cady M, Rossi RL, Silvermann ML, Piccione W, Heck TA. Gastric carcinoma, A disease in transition. Arch Surg 1989;124: 303-8.

11. Breaux JR, Bringaze W, Chappuis C, Cohn I. Adenocarcinoma of the stomach: A review of 35 years and 1710 cases. World J Surg 1990;14:580-6.

12. Louwrens HD, Kotze TJvW, Brits DA, Rossouw DJ, Falck V. Has the character of gastric cancer changed? A descriptive study of a 10-year period. S Afr Med J 1991;79:364-6.

13. Lauren $P$. The two main histological types of gastric carcinoma, diffuse and so-called intestinal type carcinoma. Acta Pathol Microbiol Scand 1965;64:31-49.

14. Muir C. Malignant diseases of the gastrointestinal tract in Singapore. Br J Cancer 1959;13:595-605.

15. Swynnerton BF, Truelove SC. Carcinoma of stomach. Br MJ 1952;9:287-92.

16. Wynder EL, Kmet J, Dungal N, Segi M. An epidemiological investigation of gastric cancer. Cancer 1963;16:1461-96.

17. Powell J, MaConkey CC. Increasing incidence of adenocarcinoma of the gastric cardia and adjacent site. Br J Cancer 1990;62:4403.

18. Lund O, Hasenkam JM, Aagaard MT, Kimose HH. Timecarcinomas of the oesophagus and cardia. Br J Surg 1989;76:13017.

19. MacDonald WC. Clinical and pathologic features of adenocarcinoma of the gastric cardia. Cancer 1972;29:724-31.

20. Webb JN, Busuttil A. Adenocarcinoma of the oesophagus and of the oesophagogastric junction. Br J Surg 1978;65:475-9.

21. MacDonald WC, MacDonald JB. Adenocarcinoma of the esophagus and/or gastric cardia. Cancer 1987;60:1094-8.

22. Kubo $\mathrm{T}$, Imai $\mathrm{T}$. Intestinal metaplasia of gastric mucosa in autopsy materials in Hiroshima and Yamaguchi Districts. (in Japanese) Jpn J Cancer Res 1971;62:49-53.

23. Nakamura K, Sugano H, Takagi K. Carcinoma of the stomach in incipient phase: Its histogenesis and histological appearance. (in Japanese) Jpn J Cancer Res 1968;59:251-8.

24. Nagakura T, Kayahara M, Ueno K, Ohta T, Konishi I, Ueda N et al. A clinicopathologic study on neural invasion in cancer of the pancreatic head. Cancer 1992;69:930-5.

25. Horn A, Dahl O, Morild I. Venous and neural invasion as predictors of recurrence in rectal adenocarcinoma. Dis Colon Rectum 1991;34:798-804. 